\title{
Barrett's esophagus: endoscopic diagnosis
}

\author{
Norihisa Ishimura, ${ }^{1}$ Yuji Amano, ${ }^{2}$ Henry D. Appelman, ${ }^{3}$ Roberto Penagini, ${ }^{4}$ Andrea Tenca, ${ }^{4}$ \\ Gary W. Falk, ${ }^{5}$ Roy K.H. Wong, ${ }^{6}$ Lauren B. Gerson, ${ }^{7}$ Francisco C. Ramirez, ${ }^{8}$ J. David Horwhat, ${ }^{9}$ \\ Charles J. Lightdale, ${ }^{10}$ Kenneth R. DeVault, ${ }^{11}$ Giancarlo Freschi, ${ }^{12}$ Antonio Taddei, ${ }^{12}$ \\ Paolo Bechi, ${ }^{12}$ Maria Novella Ringressi, ${ }^{12}$ Francesca Castiglione, ${ }^{12}$ \\ Duccio Rossi Degl'Innocenti, ${ }^{12}$ Helen H. Wang, ${ }^{13}$ Qin Huang, ${ }^{14}$ Andrew M. Bellizzi, ${ }^{15}$ \\ Mikhail Lisovsky, ${ }^{16}$ Amitabh Srivastava, ${ }^{16}$ Robert H. Riddell, ${ }^{17}$ Lawrence F. Johnson, ${ }^{18}$ \\ Michael D. Saunders, ${ }^{19}$ and Ram Chuttani ${ }^{20}$

\begin{abstract}
${ }^{1}$ Department of Gastroenterology, Shimane University Hospital, Shimane, Japan. ${ }^{2}$ Division of Gastrointestinal Endoscopy, Shimane University Hospital, Shimane, Japan. ${ }^{3}$ Department of Pathology, University of Michigan, Ann Arbor, Michigan. ${ }^{4}$ Università degli Studi and Fondazione IRCCS "Ca Granda," Milan, Italy. ${ }^{5}$ Division of Gastroenterology, University of Pennsylvania School of Medicine, Philadelphia, Pennsylvania. ${ }^{6}$ Walter Reed Army Medical Center/National Naval Medical Center, Uniformed Services University of Health Sciences, Bethesda, Maryland. ${ }^{7}$ Stanford University School of Medicine, Stanford, California. ${ }^{8}$ Mayo Clinic, Scottsdale, Arizona. ${ }^{9}$ Gastroenterology Service, Walter Reed Army Medical Center, Washington, District of Columbia. ${ }^{10}$ Columbia University Medical Center, New York, New York. ${ }^{11}$ Department of Medicine, Mayo Clinic College of Medicine, Jacksonville, Florida. ${ }^{12}$ Department of Medical and Surgical Critical Care, Unit of Surgery, University of Florence, Florence, Italy. ${ }^{13}$ Department of Pathology, Beth Israel Deaconess Medical Center and Harvard Medical School, Boston, Massachusetts. ${ }^{14}$ Department of Pathology, VA Boston Healthcare System and Harvard Medical School, West Roxbury, Massachusetts. ${ }^{15}$ Department of Pathology, Brigham and Women's Hospital, Harvard Medical School, Boston, Massachusetts. ${ }^{16}$ Pathology Department, Dartmouth Hitchcock Medical Center, Lebanon, New Hampshire.

${ }^{17}$ University of Toronto, Mount Sinai Hospital, Toronto, Ontario, Canada. ${ }^{18}$ Division of Gastroenterology \& Hepatology, University of Alabama, Birmingham, Alabama. ${ }^{19}$ University of Washington Medical Center, Seattle, Washington. ${ }^{20}$ Beth Israel Deaconess Medical Center, Harvard Medical School, Boston, Massachusetts
\end{abstract}

This collection of summaries on endoscopic diagnosis of Barrett's esophagus (BE) includes the best endoscopic markers of the extent of BE; the interpretation of the diagnosis of ultra-short BE; the criteria for endoscopic grading; the sensitivity and specificity of endoscopic diagnosis; capsule and magnifying endoscopy; narrow band imaging; balloon cytology; the distinction between focal and diffuse dysplasia; the techniques for endoscopic detection of dysplasia and the grading systems; and the difficulty of interpretation of inflammatory or regenerative changes.

Keywords: gastroesophageal junction; C\&M criteria; palisade vessels; ultrashort segment; Prague criteria; focal islands; narrow band imaging; Barrett's esophagus; endoscopic diagnosis; PPV; ACG guidelines; capsule endoscopy; PillCam; string capsule; trimodal imaging; high resolution endoscopy; chromoendoscopy; specialized intestinal metaplasia; magnification endoscopy; chromoendoscopy; NBI; specialized columnar epithelium; neoplastic progression; Vienna Classification System; 5-ALA sensitization; protoporphyrin; PpIX; confocal laser endomicroscopy

\section{Concise summaries}

- A new modification may be necessary for the Prague C\&M criteria, taking into account the technical difficulty for the definition of gastroesophageal junction (GEJ) by endoscopic landmarks. However, the reliability of the Prague criteria, evaluated by a measure of interobserver agreement for recognizing different lengths of Barrett's esophagus (BE) and the position of the GEJ indicated by the proximal margin of the gastric folds was very satisfactory.

- Ultra-short segment Barrett's mucosa (USSBE) or intestinal metaplasia (IM) at the junction is common. To put it under surveillance comes with excessive demands, and it should be ignored.

- Contrast endoscopy appears to be the most practical way to detect islands of IM after 
ablation. Endomicroscopy has potential but is less practical for this goal. Acetic acid chromoendoscopy has good potential but is not widely used. Utilizing the ACG guidelines, the positive predictive value (PPV) of endoscopically identifying BE would vary considerably depending on the number of biopsies obtained and the length of the Barrett's epithelium. Further advances in video capsule endoscopy technology, allowing for even better visualization of the $\mathrm{Z}$-line and possible tissue acquisition, could render the performance parameters of this technique favorable compared to standard upper endoscopy.

- String capsule endoscopy (SCE), allowing controlled movement within the esophagus, seems to have better accuracy in the diagnosis of BE. Narrow band imaging (NBI) has changed the approach to Barrett's in specialized centers in that it allows a more detailed view and mapping of the affected segment. It is used as an adjunct to conventional white-light endoscopy for targeted investigation of suspicious areas.

- However, trimodal imaging is still unable to be considered superior to random four-quadrant biopsies (4QB) taken with standard endoscopy with regard to detection of lesions.

- Current evidence shows that autofluorescencebased strategies are still expensive, not widely available, nonstandardized, and even in expert hands suffer from an unacceptably high false positive rate to make it viable at this time. Balloon cytology has low sensitivity but high specificity to detect low-grade dysplasia in $\mathrm{BE}$. A new type of mechanical balloon and ancillary molecular study may improve the sensitivity of this technique.

- DNA ploidy determination appears to be a powerful method for determination of cancer risk and progression in BE-related disease and can be carried out by improved image cytometry that is easier to use and more reliable than flow cytometry (FC). With histology as the gold standard, balloon cytology has been found to have low sensitivity, but reasonable to high specificity. There is a need to minimize the discrepancies in interpretation of morphologic dysplasia between Western and Japanese pathologists and to reach a uniform consensus on the nomenclature of neoplastic precursor lesions.

- Endoscopic FED gives a comparable result to that obtained with four quadrants biopsies taken every $1 \mathrm{~cm}$. High-resolution (HR) endoscopy has likely become the default standard imaging technique for best selection of biopsies and endoscopic diagnosis of dysplasia.

\section{What is the most consistent landmark for the endoscopic diagnosis of BE? What are the best markers of the distal extent of endoscopic BE by use of standard equipment?}

\author{
Norihisa Ishimura and Yuji Amano \\ amano@med.shimane-u.ac.jp
}

A reliable diagnosis of $\mathrm{BE}$ depends on the accurate endoscopic recognition of the anatomic landmarks at the GEJ and squamocolumnar junction (SCJ). To standardize the objective diagnosis of endoscopic $\mathrm{BE}$, the Prague C\&M criteria was proposed by a subgroup of the International Working Group for the Classification of Oesophagitis (IWGCO). ${ }^{1}$ In this system, the landmark for the GEJ is the proximal end of the gastric folds, not the distal end of palisade vessels, which are used to endoscopically identify the GEJ in Japan. Although the Prague C\&M criteria are clinically relevant, an important shortcoming of this system may be failure to identify short segment BE $(\mathrm{SSBE}),{ }^{2}$ a lesion that is found frequently in most Asian countries, including Japan.

Our aim was to compare the diagnostic yield for $\mathrm{BE}$ when using the gastric folds versus palisade vessels as a landmark for the GEJ, and we evaluated interobserver diagnostic concordance. ${ }^{3}$

Eighty-four endoscopists classified 30 patients with $\mathrm{BE}$ by viewing projected endoscopic photographs. The endoscopists were asked to identify the GEJ, first by using palisade vessels as a landmark and then by using the gastric folds. Endoscopists were divided into groups according to years in practice as an endoscopist, presence, or absence of board 
certification from the Japan Gastroenterological Endoscopy Society, and whether they had taken any special endoscopic training courses on GERD. The $\kappa$ coefficient of reliability was calculated for each group.

\section{Results}

Of the 30 cases, 17 had clearly visible palisade vessels, whereas the vessels could not be found in 13 cases. In 17 cases with clearly visible palisade vessels, the $\kappa$ value was 0.16 , an unacceptably low value of concordance over chance agreement. The value of trained endoscopists was significantly higher than that of nontrained endoscopists. In 13 cases where gastric folds were used as a landmark of GEJ, the $\kappa$ values were lower than those in palisade vessels criteria. Trained endoscopists also showed a higher $\kappa$ value than nontrained endoscopists. To assess the effectiveness of the systematic education and training about the Prague C\&M criteria, the same trained endoscopists were thoroughly explained this criteria, and the same test was repeated two months later. As a result, the diagnostic concordance significantly increased from 0.17 to 0.35 after the education and training about the criteria and the use of the proximal end of gastric folds as a landmark. In the evaluation by the still photographs, gastric folds showed a higher $\kappa$ value than palisade vessels as a landmark of the GEJ.

The most important weakness of proximal ends of gastric folds is that the diagnostic concordance is low in SSBE, which is difficult to identify the proximal end of gastric folds. Moreover, the position of the proximal margin of the gastric fold can easily change by altering the degree of air deflation with endoscopy (Table 1).

Palisade vessels can be found easily when the lower esophagus is adequately distended. However, the visual identification of palisade vessels may be disturbed by several factors including mucosal inflammation, dysplastic changes, and the presence of a thick double muscularis mucosa. Insufficient extension and inadequate stretch of the esophagus under conscious sedation may also disturb the identification of palisade vessels. Thus, neither of the landmarks of GEJ-gastric folds and palisade vessels - are the ideal, for different reasons, and are difficult to identify accurately and consistently in all patients with BE. ${ }^{4}$
Table 1. Technical difficulty for definition of GEJ by endoscopic landmarks

\begin{tabular}{lc}
$\begin{array}{l}\text { Endoscopic landmark } \\
\text { of GEJ }\end{array}$ & $\begin{array}{c}\text { Technical } \\
\text { difficulty }\end{array}$ \\
\hline $\begin{array}{c}\text { Proximal ends of gastric } \\
\text { folds (C\&M criteria) }\end{array}$ & $\begin{array}{c}\text { The position can easily } \\
\text { change by air deflation } \\
\text { Unable to recognize the } \\
\text { fold in cases with severe } \\
\text { atrophic gastritis }\end{array}$ \\
$\begin{array}{c}\text { Distal ends of palisade } \\
\text { vessels (Japanese } \\
\text { criteria) }\end{array}$ & inflammation and \\
& dysplastic change are \\
& present \\
& Insufficient distention of \\
& the lower esophagus \\
& under conscious \\
& sedation may disturb \\
& the identification of the \\
& vessels
\end{tabular}

\section{Conclusion}

A new modification may be necessary for the C\&M criteria, as more accurate and suitable criteria for both patients with long segment BE (LSBE) and SSBE. Also, systematic education and training are important to improve the diagnostic consistency in patients with SSBE, especially for less experienced endoscopists.

\section{Is the diagnosis of USSBE beneficial for patient care?}

Henry D. Appelman

appelman@umich.edu

To answer this question, we need to know the definition of USSBE. This seems to be the same as IM, identified by the presence of goblet cells, at the GEJ, on the columnar side of the SCJ. ${ }^{5}$ To satisfy this definition, does the SCJ have to be normal or can it be exaggerated? This is not clear. Because this junction region is so small, a tiny patch of IM in a biopsy from the junction may be either on the esophageal or on the gastric cardiac side, and there is no way to tell from the microscopic exam.

According to the 2008 American College of Gastroenterology (ACG) Practice Parameters Committee definition, $\mathrm{BE}$ is " $\mathrm{a}$ change in the distal esophageal epithelium of any length that can be 
recognized as columnar type mucosa at endoscopy and is confirmed to have IM by biopsy of the tubular esophagus." 6 This is somewhat different from the 2006 definition from the British Society of Gastroenterology in which BE is defined as "an endoscopically apparent area above the esophagogastric junction that is suggestive of Barrett's, which is supported by the finding of columnarlined esophagus on histology." "In this definition, the presence of goblet cells are not a requirement for diagnosis, presumably because limited sampling may miss them. However, USSBE does not satisfy either the American or British endoscopic requirements for the diagnosis of Barrett's mucosa, so it does not deserve to be given the Barrett name.

How common is USSBE? About $15 \%$ of people with normal-appearing SCJs have IM, so this clearly is not seen endoscopically. ${ }^{8}$ About a third or more of people with jagged, irregular, or especially prominent SCJs have IM. This irregularity or especial prominence can be seen endoscopically, so, perhaps, this may be considered to be a modification of the endoscopic requirements for Barrett's mucosa. However, if these data are correct, then it is likely that millions of people have USSBE.

Barrett's mucosa is only important because it has a cancer risk, so does USSBE have a cancer risk comparable to that of typical Barrett's mucosa? Some studies suggest that the length of columnar-lined esophagus of Barrett's mucosa is a significant risk factor in the development of dysplasia and carcinoma. One study indicates that the risk for adenocarcinoma in patients with short segment Barrett's is not substantially lower than that in long segment disease, although the results of that study did suggest a small increase in risk for neoplastic progression with increasing segment length. In this study, seven patients had IM at the junction-that is, USSBE-without endoscopic Barrett's mucosa, and no cancers were detected during over 23 person years of follow-up. ${ }^{9}$ Therefore, there seems to be no data proving that IMGEJ has a cancer risk comparable to long segment Barrett's mucosa or even short segment Barrett's mucosa. In fact, there is no data proving that USSBE has any increased cancer risk.

What is the downside for labeling a patient as having Barrett's mucosa based simply on IM at the junction? First, there is the patient's anxiety because of perceived cancer risk, which then leads to the need for repeat surveillance endoscopy and biopsy.
Insurability problems are superimposed, and, in addition, it is commonly difficult to find that IM at subsequent endoscopy and biopsy.

In summary, USSBE or IM at the junction is common. To put it under surveillance comes with excessive demands on our medical resources including endoscopists, biopsies, and pathologists to interpret the biopsies. It does not satisfy any of the definitions of Barrett's mucosa because it does not have the endoscopic findings. It does not have an established cancer risk, and it is difficult to find at subsequent endoscopies. These facts lead to this obvious conclusion: USSBE, also known as IM at the GEJ, should be ignored.

\section{What is the value of the Prague C\&M criteria for standardization of the endoscopic grading of BE and its progression over time?}

Roberto Penagini and Andrea Tenca

roberto.penagini@unimi.it

Validated endoscopic descriptions of BE are lacking. The International Working Group for the Classification of Oesophagitis (IWGCO) has recently validated criteria for the endoscopic description of BE. ${ }^{2}$ These criteria are called the Prague C\&M because they were developed during the United European Gastroenterology Week in Prague and are based on measurement of both circumferential (C) and maximal (M) extent of metaplasia (Fig. 1). In the original paper, criteria were externally validated by 29 expert endoscopists. The reliability coefficient (RC), a measure of interobserver agreement, for recognizing different lengths of $\mathrm{BE}$ ( $\mathrm{C} \& \mathrm{M}$ values), and the position of the GEJ indicated by the proximal margin of the

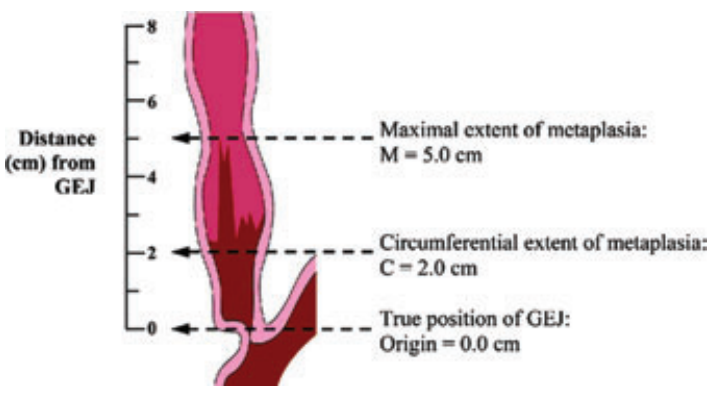

Figure 1. The Prague C\&M criteria by the International Working Group for the Classification of Oesophagitis (IWGCO). ${ }^{2}$ With permission from Elsevier. 
gastric folds were very good, ranging from 0.88 to 0.94. Recognition of $\leq 1 \mathrm{~cm}$ of BE was, however, only slightly reliable (RC of 0.21 ). Recently, criteria have also been validated by 16 gastroenterology trainees and RCs were similarly high, ranging from 0.93 to 0.97. ${ }^{10}$ These data show that the Prague C\&M criteria can reliably be used in clinical practice both by experts and trainees after adequate teaching. A recent study in Japan has highlighted the importance of training on Prague criteria and has shown that the RC of 25 experienced endoscopists for identification of the GEJ was poor when assessed without training and it improved markedly after training. ${ }^{3}$

Considering that validation of the Prague criteria has been done using video clips or still photographs, another study from Japan involving experienced endoscopists has suggested that $\mathrm{RC}$ for recognizing the position of the GEJ is less during live endoscopy than with still photographs. ${ }^{4}$ A study by Kinjo et al. has questioned the use of the proximal margin of gastric fold for identification of the GEJ in the Japanese population where gastric folds are less visible because of a high prevalence of atrophic gastritis. ${ }^{11}$ In this population, the lower limit of the palisade vessels (using HR endoscopy [HRE]) has been suggested to be a more reliable indicator. Finally, ongoing studies will assess whether the Prague C\&M criteria have an impact on overall patients' outcome.

\section{What are the means of detecting focal islands of SIM not visible at conventional endoscopy?}

Gary W. Falk

gary.falk@uphs.upenn.edu

There are two scenarios where one is trying to address focal islands of IM. The first is an attempt to detect IM within the mosaic of columnar epithelium that is characteristic of a typical BE segment. The second is to detect islands of IM after endoscopic ablation by any of a variety of different methods. The current tools available to accomplish this task are chromoendoscopy, contrast endoscopy, and confocal endomicroscopy.

Methylene blue ${ }^{12}$ is a vital stain that selectively diffuses into the cytoplasm of absorptive epithelium of the small intestine and colon. The presence of staining in the esophagus indicates the presence of
IM. However, there is no agreement on application technique in terms of the concentration, volume, and "dwell time" for methylene blue chromoendoscopy and interpretation of staining is subjective. Methylene blue chromoendoscopy ${ }^{12}$ also adds additional procedure time. A recent meta-analysis found that methylene blue chromoendoscopy resulted in no incremental yield when compared to random biopsies for the detection of IM, high-grade dysplasia, or early cancer.

On the other hand, application of acetic acid ${ }^{13}$ in conjunction with magnification endoscopy ${ }^{14}$ can enhance the detection of IM both before and after ablation therapy. However, this requires pit pattern interpretation and magnification endoscopic techniques, thereby making this method of uncertain clinical utility.

A variety of optical imaging enhancements have been developed to allow for detailed inspection of the mucosal and vascular surface patterns. $\mathrm{NBI}^{15}$ involves the placement of optical filters that narrow the band width of white light to blue light. This allows for detailed imaging of the mucosal and vascular surface patterns in BE without the need for chromoendoscopy. There are also two postprocessing software-driven systems to accomplish similar visualization (I-scan and FICE). A recent systematic review found that NBI has a sensitivity of $77-100 \%$ and a specificity of $79-94 \%$ for the detection of IM. The overall accuracy is estimated to be approximately $88-96 \%$. There are a number of unresolved issues regarding NBI, including the use of multiple classification schemes for mucosal and vascular patterns, image interpretation based on still images instead of real time endoscopy, the use of optical versus electronic zoom, and the use of study populations enriched with early neoplasia in tertiary care centers.

Confocal laser endomicroscopy $(\mathrm{CLE})^{16}$ is a new endoscopic imaging technique that allows for subsurface imaging and in vivo histologic assessment of the mucosal layer during standard white light endoscopy. It is a potential ideal small field imaging technique that optimally should be used with a "red flag" method to target image acquisition. The main goal of endomicroscopy is to distinguish neoplastic from nonneoplastic tissue in "real time" and thus provide the potential for decreased number of biopsies. However, there are also clear criteria that may distinguish IM from gastric type metaplasia at the 
time of endoscopy that may be helpful in the future. Two different platforms are available: a scope-based device that is integrated into the distal tip of the endoscope and a probe-based device that can be inserted through a standard endoscope. Both devices require administration of an intravenous fluorescence agent, fluorescein.

In summary, contrast endoscopy appears to be most practical way to detect islands of IM after ablation. Endomicroscopy has potential but is less practical and too time consuming for this goal. Acetic acid chromoendoscopy has good potential but is not widely used.

\section{What is the sensitivity-specificity of the endoscopic diagnosis of BE?}

\section{Roy K.H. Wong}

Roy.Wong@us.army.mil

This question will be rephrased to ask: "what is the positive predictive value of diagnosing Barrett's esophagus at endoscopy?" This definition would relate to endoscopies which reveal tongues or long columns of columnar appearing epithelium within the esophagus that when biopsied reveal either columnar epithelium or intestinal dysplasia.

The PPV would be calculated by the formula: true histologically proven BE positives/endoscopically suspected BE. The PPV would depend on the definition of BE. According to the British Society of Gastroenterology, ${ }^{7}$ any histologically proven colum- nar epithelium within the esophagus would be considered BE, whereas the ACG Guidelines ${ }^{6}$ define $\mathrm{BE}$ as requiring $\mathrm{IM}$ within the columnar appearing epithelium.

Utilizing these criteria, there would be a much higher PPV for BE with the British definition. For LSBE, it would be $100 \%$; whereas for SSBE, it would probably be greater than $95 \%$ with difficulty differentiating a very short segment of BE also known as cardia IM (CIM), esophagogastric junction specialized IM (EGJ-SIM), and ultra-short segment BE from a normal SCJ.

Utilizing the ACG guidelines, the PPV of endoscopically identifying BE would vary considerably depending on the following factors: (1) the number of biopsies obtained and (2) length of the Barrett's epithelium ${ }^{17}$; whether the biopsies were obtained from areas of esophagitis or visually uninflamed columnar epithelium.

Studies by Harrison et al. ${ }^{17}$ indicate that the more biopsies obtained, the greater the likelihood of identifying BE. For practical purposes, the optimal number of biopsies that should be taken is eight per procedure with a likelihood of identifying IM in $67.9 \%$. If only four biopsies are obtained, $34.7 \%$ would be positive for IM. Another factor that is associated with a higher yield is the length of the columnar epithelium. ${ }^{18}$ As the length of the tongue or column of columnar epithelium increases, the likelihood of identifying IM will also increase. In histological studies, the IM was proximal, located mainly next to the SCJ with fundic and cardia cells noted

Table 2. Positive predictive value of identifying BE with SIM

\begin{tabular}{|c|c|c|c|c|}
\hline Author & $n$ & EGJ-SIM (prevalence) & SSBE (PPV) & LSBE (PPV) \\
\hline Winters et al. ${ }^{76}$ (Gastro) & 97 & & & $50 \%$ \\
\hline Cameron et al..$^{77}$ (Gastro) & 27 & & $37 \%$ & \\
\hline Johnston et al. ${ }^{78}$ (AJG) & 172 & $7 \%$ & $9.4 \%$ & $100 \%$ \\
\hline Weston et al. ${ }^{79}$ (AJG) & 237 & & $48 \%$ & \\
\hline Trudguill et al. ${ }^{80}$ (Gut) & 120 & $18 \%$ & & \\
\hline Pereira et al. ${ }^{81}$ (Gut) & 75 & $25 \%$ & $61.3 \%$ & \\
\hline Hackelsberger et al. ${ }^{82}$ (Gut) & 423 & $13.4 \%$ & $44 \%$ & $88 \%$ \\
\hline Eloubeidi et al. ${ }^{18}$ (AJG) & 146 & & $25 \%$ & $55 \%$ \\
\hline Hirota et al. ${ }^{83}$ (Gastro) & 889 & & $54 \%$ & $73 \%$ \\
\hline Harrison et al. ${ }^{17}$ (AJG) & 296 & & \multicolumn{2}{|c|}{$\begin{array}{l}\text { Mixed group }(1-11 \mathrm{~cm}, \text { mean }=4 \\
\mathrm{cm}) 16 \mathrm{Bx}=100 \%, 8 \mathrm{Bx}= \\
67.9 \%, 4 \mathrm{Bx}=34.7 \%\end{array}$} \\
\hline
\end{tabular}


in the mid- and proximal portion of the columnar lined esophagus. Finally, biopsies obtained from esophagitis had a lower likelihood of identifying BE by $12 \% .{ }^{19}$

The calculated PPV for SSBE ranged from 9.4\% to $61.3 \%$ and for LSBE $50 \%$ to $100 \%$ for LSBE (Table 2).

\section{Is capsule endoscopy an accurate enough tool for BE screening in clinical practice?}

\section{Lauren B. Gerson}

lgerson@stanford.edu

Wireless video capsule endoscopy (VCE) was approved by the Food and Drug Administration in 2001 as an adjunctive aid for the detection of small bowel disorders. Because patients ingest the capsule in the standing position and the small bowel VCE captures two frames per second, the traditional VCE often does not capture images of the esophagogastric junction. Developed in 2004, the esophageal capsule, or PillCam ESO (Given Imaging, Ltd., Duluth, GA, USA), captures 14 frames per second whereas the patient ingests the capsule in a supine position and then gradually resumes the sitting position during a 5-minute period. ${ }^{20}$ Usage of the first generation PillCam ESO demonstrated excellent sensitivity and specificity for the detection of erosive esophagitis and BE in a preliminary study of 106 patients (93 with GERD, 13 with BE). ${ }^{21}$ However, the results may have been biased by the usage of a post hoc adjudication committee and $\mathrm{BE}$ was not confirmed by biopsy. A second blind prospective trial demonstrated a sensitivity of $67 \%$ for BE detection comparing PillCam ESO to standard esophagogastroduodenoscopy (EGD). ${ }^{22}$ Using these performance parameters, two cost-effectiveness models were unable to conclude that the usage of esophageal capsule endoscopy (ECE) was cost-effective in the screening of BE. ${ }^{23,24}$ The main reason for these findings were that the detection rates of $\mathrm{BE}$ were modeled to be $70 \%$ for ECE compared to $85 \%$ with EGD, in addition to a $50 \%$ poor visualization rate of the EGJ, which would lead to an upper endoscopic examination and increase the cost of the EGD arm.

A second generation esophageal capsule, ESO2, was released by Given Imaging in 2007 with a $30 \%$ increase in the frame capture rate from 14 to
18 frames per second, advanced optics with three lenses instead of one lens, and expansion of field of view from $140^{\circ}$ to $169^{\circ} .{ }^{25}$ To maximize visualization of the EGJ and reduce the presence of bubbles, the standardized ingestion protocol (SIP) was published by Gralnek et al. and included having the patient lie on his/her right side during capsule ingestion while sipping $5-10 \mathrm{ml}$ of water every 30 seconds. ${ }^{25}$ A subsequent clinical trial in 28 subjects using the SIP protocol and ESO-2 demonstrated visualization of the Z-line in $75 \%$ of subjects, and sensitivity of $100 \%$ with specificity of $74 \%$ for BE detection. ${ }^{26}$ The agreement between ESO-2 and EGD for description of the $\mathrm{Z}$-line was $86 \%(\kappa=0.68)$.

A 2009 meta-analysis ${ }^{27}$ including nine studies with 618 patients undergoing primarily the first generation ECE demonstrated a pooled sensitivity and specificity of ECE for BE detection of $77 \%$ and $86 \%$ compared to $78 \%$ sensitivity and $90 \%$ specificity for EGD. Usage of SCE in one study demonstrated sensitivity of $78 \%$ with specificity of $83 \% .{ }^{28}$ The authors concluded that upper endoscopy should remain the preferred modality in the evaluation of patients with suspected BE.

Therefore, although the usage of ECE has been demonstrated to be an acceptable screening modality for the presence of esophageal varices, ${ }^{29}$ it is not possible in most regions of the United States to obtain insurance authorization in order to perform an ECE study in a patient for evaluation of GERD and/or screening for BE. Given the superior performance parameters with usage of the ESO-2 and SIP protocol, however, further clinical studies may be able to add to the encouraging results from the initial study using ESO-2. In addition, further advances in VCE technology allowing for even better visualization of the Z-line and possible tissue acquisition, could render the performance parameters of VCE favorable compared to standard upper endoscopy.

\section{Should SCE, allowing controlled movement within the esophagus, have better accuracy in the diagnosis of BE?}

\section{Francisco C. Ramirez \\ FRami36715@aol.com}

The major drawbacks of the ECE device in its current form that may affect the accuracy of the images and thus the diagnosis of an esophageal 
Table 3. Performance characteristics of string capsule endoscopy (SCE) for the diagnosis of Barrett's esophagus when using esophagogastroduodenoscopy (EGD; visual) and intestinal metaplasia (IM) as the gold standard

Sensitivity Specificity PPV NPV Accuracy

\begin{tabular}{llllll}
\hline SCE (visual) & $78 \%$ & $89 \%$ & $86 \%$ & $83 \%$ & $84 \%$ \\
EGD (IM) & $87 \%$ & $69 \%$ & $59 \%$ & $91 \%$ & $75 \%$ \\
SCE (IM) & $94 \%$ & $79 \%$ & $79 \%$ & $96 \%$ & $84 \%$
\end{tabular}

PPV, positive predictive value; NPV, negative predictive value.

condition, $\mathrm{BE}$ in this case, include (1) esophageal transit time, which often is too short and in about $5 \%$ may be too prolonged, but, more importantly, is unpredictable with reported ranges from 1 to $1,678 \mathrm{sec}$; (2) the challenge of the Z-line visualization and its relationship to the gastro-esophageal junction; (3) the potential and frequent interference of the images with bubbles and secretions and the inability to clean the lens(es); and (4) the "snapshot" phenomenon that refers to the inherent inability to reexamine any area of interest multiple times and when in doubt. The data regarding the performance characteristics of wireless capsule endoscopy in the diagnosis of BE have been reported recently in the form of a meta-analysis. ${ }^{32}$ The pool sensitivity and specificity were found to be $77 \%$ and $86 \%$, respectively for all studies analyzed. The pooled sensitivity and specificity of capsule endoscopy when EGD was used as the gold standard were $78 \%$ and $90 \%$, and when IM was used as the gold standard, were $78 \%$ and $73 \%$, respectively (the low specificity was thought to be the result of a study published in abstract form only). SCE was designed by attaching a tethering device (composed of strings, or strings with a sleeve) to the small bowel or the esophageal capsule devices for circumventing the drawbacks outlined above and its feasibility initially tested for $\mathrm{BE}^{30}$ and esophageal varices. ${ }^{31}$ The control of the capsule device through strings converts a physiological-dependent into an operatordependent procedure. The addition of the real time viewer makes the procedure easier and more efficient. An additional feature allows its retrieval and, after discarding the tethering device and sterilization or high-grade disinfection, to reuse the capsule and thus render the procedure cost-effective.

The data of SCE of 100 consecutive patients ${ }^{28}$ referred for screening of BE and when using IM as the gold standard are summarized in Table 3 .

So, going back to the question: should SCE, allowing controlled movement within the esophagus, have better accuracy in the diagnosis of BE? The answer is not only that it should, but, it does. However, the data need to be validated.

\section{What attitude should be adopted when there are no visible abnormalities in a patient?}

\section{John David Horwhat \\ John.david.horwhat@us.army.mil}

One can appreciate that the ability to diagnose Barrett's when encountering a columnar-lined esophagus at endoscopy is not a straightforward task, so what is meant by "no visible abnormalities?" First, we must acknowledge that what we see when we perform endoscopy is greatly influenced by a host of factors, including our training and the prejudices, biases, and experiences that we have had in our practices (Table 4 ). Too often, what we see is

Table 4. Comparison of imaging techniques

\begin{tabular}{lllll}
\hline Modality & Availability & Cost & Ease of use & Utility \\
\hline Indigo carmine & +++ & + & +++ & $++/+++$ \\
Methylene blue & +++ & + & ++ & $+/++$ \\
Acetic acid & +++ & + & +++ & +++ \\
High definition endoscopy & ++ & ++ & +++ & +++ \\
Narrow band imaging (zoom) & $+/++$ & ++ & + & +++ \\
Autoflourescence imaging & + & +++ & + & ++ \\
Trimodal endoscopy & + & & + l+ & ++ \\
\hline
\end{tabular}


what we expect to see, and we can certainly miss subtle abnormalities if our eyes are not calibrated or trained to detect them. In fact, what is not visible or conspicuous to one endoscopist may be a glaring abnormality to another. One needs only to compare adenoma detection rates between colonoscopists to gain an understanding of how similar physicians can achieve different outcomes.

Further, we should acknowledge that looking is not the same as seeing, and you need to know what you are looking at. This includes an understanding of the appearance and proper interpretation of findings at simple white light endoscopy. Very subtle changes in the surface texture or color may be overlooked if one fails to clean surface mucus with proper irrigation, take the time to insufflate or let the lumen quiet after peristalsis. Just as one might overlook a flat adenoma in the colon, we need to be aware for subtleties when examining patients with a columnar-lined esophagus. Clearly, if one has the ability to perform surface enhancement with acetic acid and/or magnify the image, the endoscopist must have a complete understanding of, and ability to, interpret both the normal and abnormal vascular and pit patterns of the mucosa before stating there are no visible abnormalities.

Next, one must know how best to employ existing technologies to see the mucosa. We realize that not all of us currently have or will be able to justify the purchase of equipment to perform advanced imaging techniques. And even if we do have these scopes in our centers, not all of us will have been properly trained in the interpretation of images taken with narrow band or multiband endoscopy or the application of red flag technology such as autofluorescence to avoid high false positive rates. Certainly, we cannot expect all the world's Barrett's patients to only be treated at centers of excellence that do have this capability. So what can the rest of us do?

Indigo carmine, as shown by a multicenter study of 56 patients, had a sensitivity and specificity in the $83-88 \%$ range and negative predicitive value (NPV) of $98 \%$ for HGD. ${ }^{32}$ A recent meta-analysis reviewing work done with methylene blue concluded that there is really no significant incremental yield over random biopsies for the detection of SIM or dysplasia with this agent. ${ }^{15}$ Acetic acid scores favorably in being cheap, readily available and easy to use. A recent study from Longcroft-Wheaton et al. demonstrated a $95.5 \%$ sensitivity and a near perfect correlation with final histology $(r=0.98)$ when using acetic acid for guiding targeted biopsies using HR white light endoscopy. ${ }^{33}$ HRE is hard to describe by itself because the bulk of the literature speaks to HR NBI or HR acetic acid and not merely the incremental gain of HR compared to standard endoscopy.

To consider narrow band and multiband imaging, we need to acknowledge that the published work with this technology is almost entirely with magnification using 240 series Olympus endoscopes not available in the United States. With autofluorescence and trimodal endoscopy, we shift toward modalities that are still very much confined to a few centers of excellence-namely Amsterdam; Nottingham, UK; Jacksonville, Florida; and Rochester, Minnesota. Even within these centers, there remains difficulty in finding the best application and interpretation of this technology. Experts from these centers convened to determine how to reduce false positives seen with autoflourescence by trying to determine what endoscopic features were most predictive of early neoplasia in AF-positive areas. The best they could accomplish was moderate agreement with a $\kappa$ ranging from 0.49 to $0.56 .^{34}$ This statement is not meant to impugn their efforts or the technology, but merely to show that we are still quite far away from realizing the best way to employ this technology.

So, what current guidance can we take from the experts? A recent multicenter study has demonstrated that trimodal imaging is still unable to be considered superior to random $4 \mathrm{QB}$ taken with standard endoscopy with regard to detection of lesions. ${ }^{35}$ This study, from the group of four centers of excellence previously mentioned, used an HR trimodal Olympus system versus standard endoscopy with an Olympus 140 or 160 series instrument. Although the targeted yield of endoscopy was improved with autofluorescence, the problem of false positives remained, and even using NBI to try and reduce the false positives was problematic in that $17 \%$ of HGD and cancer lesions were erroneously classified as nonsuspicious by NBI. In addition, a study from the Weisbaden, Germany group studied high- and low-risk Barrett's groups with HR acetic acid targeted biopsies versus standard 4QB. ${ }^{36}$ Interestingly, although the targeted exams were superior, their final statement was that they do not advocate abandoning 4QB outside of 
a setting such as exists within their highly specialized center with rigorous quality control measures in place.

In summary, the attitude that should be taken for the patient with no visible abnormalities is one of high-quality white light endoscopy. Ideally, we would all like to have HR as our standard instruments, but while we wait for this, we should at least aim to enhance our knowledge of the interpretation of normal-at a minimum, teaching ourselves and our trainees what the normal and abnormal pit and vascular patterns look like. One should learn how to optimally use the technology you currently have, but if not fortunate enough to have enhanced imaging capability, surface enhancement with cheap, readily available materials like acetic acid can be used. The current evidence shows that autofluorescence-based strategies are still too expensive, not widely available, nonstandardized, and even in expert hands suffer from an unacceptably high false positive rate to make it viable at this time. That leaves the majority of us in the United States with learning how to interpret unmagnified NBI images, learning how to better appreciate subtleties for targeted biopsies and remembering to always, always, always, take fourquadrant random biopsies to make sure we do not miss the forest for the trees.

\section{What can be expected from magnifying endoscopy associated with NBI for the detection of SIM in BE?}

Charles J. Lightdale

cj118@columbia.edu

\section{High-resolution white light endoscopy}

Modern endoscopes use HR digital systems using charge-coupled devices (CCD) in the endoscope tip with greater than 1,000,000 pixels compared to the 300,000 of prior standard endoscopes. Combined with high-definition television monitors with 1,080 scanning lines compared with the standard analog 576 lines, HRE provides an extraordinary clear and detailed moving image. ${ }^{37}$

\section{Magnification endoscopy}

HREs also are generally equipped with an electronic zoom system that provides some magnification, routinely to $1.5 \times$. However, magnification endoscopes are available using optical zoom system with mechanically adjusted lens systems for much greater magnification without loss of resolution. The difficulty is that focal length decreases with increasing magnification, which can make it difficult to keep images in focus even with the use of transparent endoscope caps to stabilize the target tissue. The maximal efficiency of these systems is in combination with chromoendoscopy. ${ }^{38}$

\section{Optical chromoendoscopy}

Optical chromoendoscopy sometimes called virtual image enhanced endoscopy provides an instant "virtual" contrast applied with the push of a button. Thus, optical chromoendoscopy offers a fast clean alternative to dye-spraying or physical chromoendoscopy. The Olympus system called NBI uses filtered light for this effect, whereas other systems use digital postprocessing. ${ }^{37,38}$

\section{Narrow band imaging}

In this system, filters alter the illuminating light, decreasing the red component, and allowing only narrow bands of blue (390-445 $\mathrm{nm}$ ) and green light $(530-550 \mathrm{~nm})$ to illuminate the mucosal surface. These short wavelengths penetrate the mucosa only superficially, improving the view of mucosal surface patterns and highlighting vasculature. ${ }^{15}$

\section{$\mathrm{NBI}$ and magnification in $\mathrm{BE}$}

Nondysplastic specialized IM (SIM) under NBI and magnification shows a regular tubular, ridged, or villous pattern with branching elongated vessels. Norimura et al. recently described an appearance of characteristic "light blue crests" in a beautifully illustrated article. ${ }^{39}$ Mannath et al. recently published a meta-analysis using NBI for detection of SIM, finding a high sensitivity of 0.95 in six pooled studies, whereas specificity was low at $0.65 .{ }^{40} \mathrm{Mag}$ nification with NBI has improved visualization of the squamo-columnar junction (Z-line) and small tongues of SIM in very short BE, and has helped detect small islands of SIM for biopsy or ablation.

\section{Can association of NBI with HRE lead to a change in the current practice of random biopsies in Barrett's patients?}

Kenneth R. DeVault

devault.kenneth@mayo.edu

In the United States, the diagnosis of BE requires the endoscopic appearance of columnar mucosa extending into the tubular esophagus along with 
histological confirmation of specialized columnar epithelium (SCE) in biopsies from that mucosa. On the other hand, many patients with the appearance of short segment BE, and even some with what appears to be long segment BE, will not have SCE on random biopsies. ${ }^{41}$ Whether this is due to the patient not having BE or whether it represents sampling error has not been clear. In addition, in regards to finding dysplasia, some patients will have dysplasia on one endoscopy and not on subsequent exams, ${ }^{6}$ which also could be due to sampling error.

Among the several methods of enhancing the mucosal view, NBI is simple, widely available, and may improve the yield of esophageal biopsies for both SCE and dysplasia. ${ }^{42}$ Based on these and other data, we would suggest that NBI does increase the yield of SCE over random biopsies, but would still do a similar number of biopsies as have been suggested for nontargeted exams (at least four biopsies for each 2 $\mathrm{cm}$ of Barrett's appearance).

Difficulties in finding dysplasia in a patient with $\mathrm{BE}$ remains a challenging proposition. A study where patients underwent both standard resolution endoscopy and HR/NBI was recently reported by Wolfsen et al. ${ }^{43}$ In that study, NBI and standard endoscopy found the same grade of dysplasia in $82 \%$ of patients, but a higher grade of dysplasia was noted $18 \%$ of patients with combined HR/NBI. In no cases did standard endoscopy find a higher grade of dysplasia than HR/NBI and there were less biopsies required in the HR/NBI study. This study has resulted in our center suggesting a careful HR/NBI evaluation of all BE patients with targeted biopsies. We still add additional random biopsies in most cases to equal at least the total minimal number suggested in the so-called Seattle protocol (four-quadrants for every $1 \mathrm{~cm}$ ).

In summary, NBI has changed the approach to Barrett's in specialized centers in that it allows a more detailed view and mapping of the affected segment. Whether this change in practice will be widely adopted remains to be seen.

\section{Can NBI be considered a tool for routine clinical practice?}

Giancarlo Freschi, Antonio Taddei, Maria Novella Ringressi, Vega Ceccherini, Francesca Castiglione, Duccio Rossi Degl'Innocenti, and Paolo Bechi giancarlo.freschi@unifi.it
When NBI was introduced, endoscopists were fascinated by the possibility of detecting the pathology in real time by simply pressing a switch, such as taking a picture. In 2006, Sharma et al. ${ }^{44}$ published an editorial with a compelling title, "Barrett's esophagus - see more, biopsy less!" The question in the title obviously concerns the diagnosis of GERD, and its pathological consequences, in normal endoscopy units rather than highly specialized BE centers.

To compare our performances to the data presented in literature and to answer the question, we analyzed 200 consecutive EGDS performed in GERD patients and evaluated the effectiveness of NBI with respect to:

- recognition of esophagitis cases (distinguishing NERD from true esophagitis);

- detection of Barrett's metaplasia; and

- detection of esophageal dysplasia (low or high grade) or adenocarcinoma. All the images were correlated with histology results.

Concerning recognition of esophagitis, previous studies have found that fewer than $40 \%$ of GERD patients have positive upper endoscopy findings. The utility of the NBI magnification system as a diagnostic tool for GERD has been explored increasingly focusing whether the changes on observed mucosal and vascular patterns correlate with histology. Sharma and Fock found that a significantly higher proportion of GERD patients had changes easy to observe using NBI, such as:

- the number, dilation, and tortuosity of intrapapillary capillary loops (IPCL);

- the presence of microerosions;

- an increase in vascularity at the SCJ; and

- columnar islands in distal esophagus.

Our experience of using NBI to diagnose erosive esophagitis, observing the parameters previously described, in 162 consecutive EGDS performed on patients with clinical symptoms indicating GERD, reveals that the improvement in diagnosis of esophagitis after NBI observation compared with simply white light observation was of nearly $25 \%(P<0.01)$. However, to date, there is still a need for prospective randomized controlled trials to validate the mucosal and vascular patterns seen in the above studies. 


\section{Detection of Barrett's metaplasia}

Theoretically, NBI has the advantage of allowing the endoscopist to make an in vivo diagnosis, without the need for random biopsies. In recent years, several studies ${ }^{44-46}$ have led to different classifications of patterns suggesting IM. These studies have described and classified patterns of mucosal and vascular morphology in BE based on magnification endoscopy associated with NBI, focusing on the recognition of IM in columnar-lined esophagus. Unfortunately, the classifications proposed in these studies consist in many different categories hampering implementation of NBI in daily practice. Some classifications use up to six categories for mucosal patterns, and others also require the description of the vascular pattern in multiple categories. In other words, although the available results from the studies describing the changes seen under NBI endoscopy for BE appear promising, the lack of a sufficiently validated and standardized classification scheme is one of the main limitations to the diffusion of the use of NBI for detecting $\mathrm{BE}$ and dysplasia. To improve the practicality of NBI in BE, some authors have proposed a more simplified classification in which only the (ir)regularity of the mucosal and vascular patterns are described. Our group used this simple classification for detection of $\mathrm{BM}$ in clinical practice. We had 89 NBI images for 38 patients in areas indicating columnar metaplasia from white light observation. The results were a good sensitivity (90\%), but only $78 \%$ for specificity with $83 \%$ for PPV (Fig. 2, left).

\section{Diagnosis of dysplasia or neoplasia}

The current practice of endoscopic surveillance in patients with $\mathrm{BE}$ has limitations. Biopsies are performed randomly and sample only $4 \%$ to $6 \%$ of the surface area of the metaplastic epithelium. Preliminary results from a randomized, controlled crossover showed that, as compared with a strategy of performing 4QB every $2 \mathrm{~cm}$, the use of targeted biopsies with narrow-band imaging identified similar proportions of patients with metaplastic lesions (85\% with each procedure) and neoplastic lesions (71\% with targeted biopsies and 55\% with 4QB, but involved fewer biopsy specimens per procedure [3.6 versus 7.6, $P<0.001]$ ]). ${ }^{44,51}$ But this is not the case when identifying dysplasia or carcinoma in $\mathrm{BE}$ patients through a simple endoscopy procedure, so that many experts conclude that it is questionable whether magnification endoscopy in combination with NBI at this time is ready to replace histological sampling for differentiating dysplastic from nondysplastic Barrett's tissues. Also, for the detection of early Barrett's neoplasia, only two studies with conflicting results have investigated NBI for this indication. Both studies, however, have considerable drawbacks that may impede their validity. Our limited experience confirms the uncertainty concerning NBI's potential in diagnosis of dysplasia (sensibility: $80 \%$; specificity: 78\%; PPV: 60\%; Fig. 2, right).

\section{Conclusion}

The available studies regarding the use of NBI in detecting GERD, SIM, and dysplasia or neoplasia appears promising and, presently, NBI is used as an adjunct to conventional white light endoscopy for targeted investigation of suspicious areas.

The main limitations of the NBI system include:

- the learning curve associated with the new technology (the reported studies have been performed in very high-risk BE patients by endoscopists very experienced in the care of such patients, but the widespread applicability of this technique is unknown);

- the lack of sufficiently validated and standardized classification schemes for the NBI patterns observed in various conditions; and
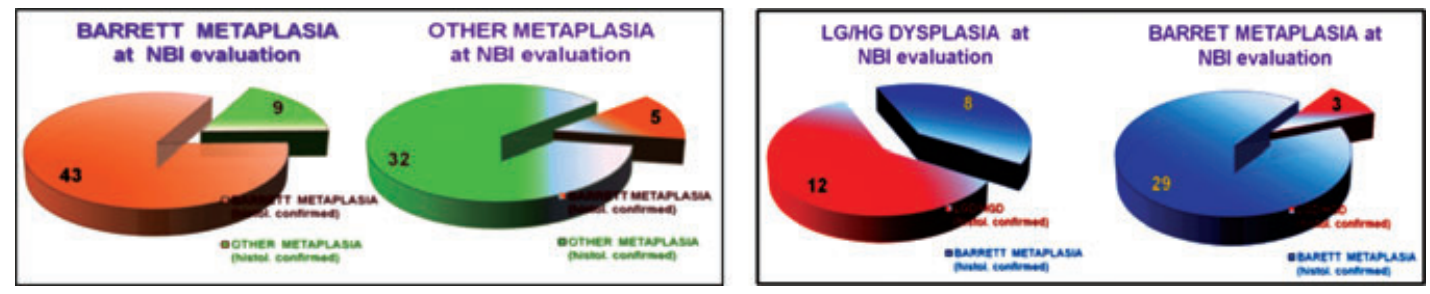

Figure 2. Personal data: results in NBI endoscopic detection of Barrett's metaplasia. 
- the limited number of randomized controlled trials investigating NBI compared with conventional white light endoscopy.

Further large-scale studies are also required to address these limitations before NBI can be recommended as a primary method of screening or follow-up for Barrett's esophagus in routine practice.

\section{What is the sensitivity-specificity of balloon cytology in detecting low-grade dysplasia?}

\section{Helen H. Wang}

hwang@bidmc.harvard.edu

Although it is a wonderful idea to screen with nonendoscopic balloon cytology for dysplasia in patients with BE, data on this subject are limited by the fact that most studies of balloon cytology were conducted in high-risk regions of China for esophageal squamous cell carcinoma. In addition, the diagnosis of low-grade dysplasia in BE is controversial even on histology as cases are often overdiagnosed and its prevalence is underestimated. ${ }^{47}$

With histology as the gold standard, balloon cytology has been found to have low sensitivity $(20-55 \%)$, but reasonable to high specificity (66-100\%). ${ }^{48-50}$ The higher sensitivity results were based on studies that involved using a new mechanical balloon for $50 \%$ of the study subjects, which apparently increased cellular yield and thus sensitivity. ${ }^{49}$

Despite its low sensitivity when compared to histology, based on an outcome/follow-up study, ${ }^{51}$ balloon cytology was useful for risk stratification of esophageal cancer, including glandular lesions. Ancillary molecular studies on cytologic specimens have been investigated to determine its value. When appropriate probes, such as for 8q24 (C-MYC), 9p21 (P16), 17q12 (HER2), and 20q13, were used, fluorescence in situ hybridization (FISH) indeed significantly improved the sensitivity in the detection of dysplasia in patients with BE. ${ }^{52}$

In summary, balloon cytology has low sensitivity but high specificity to detect low-grade dysplasia in BE. New types of mechanical balloons and ancillary molecular study may improve the sensitivity of balloon cytology.

\section{Despite the promising value of $F C$ in detecting aneuploidy of G2/tetraploidy on cytological specimens, cost, and restricted availability of this technique represent limitation of its wide clinical use}

\section{Qin Huang \\ Qin.huang@va.gov}

FC uses laser technology to measure the DNA content of cells in suspension that pass through a laser beam. The reliability of this method for detection of DNA aneuploidy associated with Barrett's esophagus and Barrett's esophageal adenocarcinoma (BEA) has been questioned for its low sensitivity and poor reproducibility. In biopsies of Barrett's esophagus, DNA aneuploidy detected by FC was considered to be a better marker than histology for assessing prognosis by some investigators, ${ }^{53}$ a conclusion that could not be confirmed by others. $^{54}$

In BEA, conventional karyotyping and comparative genomic hybridization studies have showed that aneuploidy with widespread chromosomal and DNA abnormalities are present in almost all BEA tumors. However, previous reports using FC have shown that up to $31 \%$ of BEA cases were diploid and negative for DNA aneuploidy. ${ }^{53-56}$ For example, in one recent study by standard FC in archival formalin-fixed, paraffin-embedded resection tissues with histopathology-confirmed 42 BEA tumors, ${ }^{55}$ FC detected DNA aneuploidy in only $71 \%$ of cases; the remaining $29 \%$ were diploid and negative for aneuploidy. In the cases with DNA aneuploidy, FC showed the qualitative evidence of DNA aneuploidy and failed to identify severe aneuploidy and high DNA heterogeneity fractions in $32 \%$ of cases and $9 \mathrm{~N}$ exceeding rates in $51 \%$. These parameters are the markers for high risk of cancer progression and were missed by FC. In addition to the low sensitivity of DNA aneuploidy detection and failure of identification of high-risk cases with DNA ploidy abnormalities, the reproducible rate of DNA ploidy by FC in different parts of the same tumor was only $50 \% .{ }^{55}$ This apparent sampling error is not acceptable clinically.

This poor reliability of FC in detection of DNA aneuploidy in BEA results from intrinsic technical errors due to inclusion of all cells in suspension into calculation of DNA content. Another source of errors is biopsy sampling, a problem associated 
with all biopsies. By FC, the cell suspension sample should have at least $20 \%$ of neoplastic cells with a total number of over 10,000 cells for an effective analysis. ${ }^{56}$ This requirement may not be practical for FC to meet in a small biopsy tissue sample.

In conclusion, recent data indicate that DNA ploidy analysis by FC in a biopsy or resection tissue specimen is qualitative, insensitive, poorly reproducible, and lacks quantitative information such as heterogeneity index and $9 \mathrm{~N}$ exceeding fractions for disease progression. These shortcomings along with technical flaws and high cost prevent its wide clinical applications. However, DNA ploidy determination appears to be a powerful method for determination of cancer risk and progression in Barrett esophagusrelated disease and can be carried out by improved image cytometry that is easier to use and more reliable than FC. ${ }^{57}$

\section{What is unstable aneuploidy? Is it a marker for neoplastic progression?}

\author{
Andrew M. Bellizzi \\ abellizzi@partners.org
}

\section{What is aneuploidy?}

Aneuploidy, most simply put, refers to an abnormal number of chromosomes. The aneuploid phenotype reflects genomic, and more specifically chromosomal, instability and is commonly observed across the broad range of human tumors. Although the proximate cause of aneuploidy is abnormal mitotic segregation, the ultimate causes are poorly understood. In addition to being a biomarker for neoplastic progression, aneuploidy may be mechanistically involved, by increasing the dosage of oncogenes, decreasing the dosage of tumor suppressor genes, and by serving as a source of genetic diversity on which natural selection acts. ${ }^{58}$ Aneuploidy is assessed by DNA FC and image cytometry on tissue sections.

\section{Aneuploidy and neoplastic progression in BE}

Aneuploidy represents one of the best-studied and most powerful predictors of neoplastic progression in BE. Reid et al. (Seattle Barrett's Esophagus Research Program) found that $9 / 13$ (69\%) patients with aneuploid or increased G2/tertraploid populations on initial FC developed high-grade dyspla- sia (HGD) or adenocarcinoma (AdCa) on follow up (mean 34 months). This included two patients with biopsies initially negative for dysplasia and five patients in the indefinite/low-grade dysplasia category. In contrast, $0 / 49(0 \%)$ patients without a flow abnormality progressed to HGD or AdCa. ${ }^{53}$ In a larger study from the same group, constituting their experience with a prospective surveillance cohort of 307 patients, 42 of whom developed cancer, Rabinovitch et al. reported the relative risk (RR) of aneuploidy for the development of AdCa at 5.9. They also showed that the distribution of aneuploidies is bimodal, with "triploid," and "near-diploid" aneuploidies. "Triploid" aneuploidy, which they defined as a DNA content $>2.7 \mathrm{~N}$, is an especially high-risk lesion with an RR of 9.5. ${ }^{59}$

\section{What is unstable aneuploidy?}

The term "unstable aneuploidy" is ill-defined, and in fact, a PubMed search for the terms "unstable aneuploidy" and either "esophagus" or "Barrett's" returns three results, only one of which actually uses the term "unstable aneuploidy" in the manuscript text. Although Yu et al. do not formally define "unstable aneuploidy," they refer to the "appearance of newer clones of severer aneuploidy" and state that "increased scatter of cells, including elevation of the $5 \mathrm{~N}$ exceeding fraction may represent unstable aneuploidy." They report the results of high-fidelity DNA histograms (image cytometry on formalinfixed, paraffin-embedded tissue sections) in normal controls (nl), BE, negative for dysplasia (neg), low-grade dysplasia (LGD), HGD, and AdCa. Data for each case include ploidy, heterogeneity index (a measure of the "scatter of cells"), and $5 \mathrm{~N} \mathrm{ex-}$ ceeding fraction. They found "markedly elevated" heterogeneity index values and $5 \mathrm{~N}$ exceeding fractions $>5 \%$ in $0 \%$ of $\mathrm{nl}, 5 \%$ of neg, $32 \%$ of LGD, $50 \%$ of HGD, and $88 \%$ of AdCa. Interestingly, $69 \%$ of neg cases demonstrated at least mild aneuploidy. ${ }^{60}$

\section{Is unstable aneuploidy a marker of neoplastic progression?}

Aneuploidy is common in BE and can be seen in cases even without dysplasia. It would be of interest to learn the follow up on the cases in the Yu et al. study negative for dysplasia but with "severe" DNA content abnormalities. From the Seattle Barrett's Cohort, it is clear that aneuploidy is a marker 
for neoplastic progression and that not all aneuploidies confer the same risk. Although the concept of "unstable aneuploidy" is ill-defined, it can be conceived of as "progressive aneuploidy" or "aneuploidy conferring increased risk." Yu et al.'s cross-sectional study was not positioned to answer the earlier question, which remains a testable hypothesis.

\section{How should extent of dysplasia be histologically defined? How should precise distinction between "focal" and "diffuse" dysplasia be made?}

\section{Mikhail Lisovsky and Amitabh Srivastava \\ Mikhail.Lisovsky@Hitchcock.org}

Detection of dysplasia in $\mathrm{BE}$ is the current gold standard for identification of patients at high-risk for progression to esophageal adenocarcinoma (EA). However, the natural history of BE patients with low-grade dysplasia (LGD) and high-grade dysplasia (HGD) remains uncertain and the individual risk of progression to EA is difficult to quantify. Extent of dysplasia may correlate with the risk of progression to EA, but testing of this hypothesis is compounded by the absence of a uniformly accepted method for evaluation of the extent of dysplasia. Any proposed method for measuring extent of dysplasia in BE must be objective and reproducible, in order for it to be validated across different studies, and be easily applicable in routine clinical practice.

Extent of dysplasia in $\mathrm{BE}$ as a risk factor for EA has been evaluated in only a few studies, thus far, using variable definitions. ${ }^{61-63}$ The study by Buttar et al. ${ }^{61}$ defined focal HGD as one focus of $\leq 5$ dysplastic crypts in one fragment and diffuse HGD as $\geq 5$ dysplastic crypts in one fragment or more than one fragment with HGD. ${ }^{61}$ The primary endpoint was a histologic diagnosis of EA on follow-up. Diffuse HGD had a 3.5-fold increase in the risk of progression to EA compared to focal HGD, when adjusted for endoscopic nodularity. Endoscopic nodularity was present in $34 \%$ of diffuse HGD, only $6 \%$ of focal HGD and was associated with a 2.5 times higher risk of EA when adjusted for extent of dysplasia. Thus, extent of HGD and endoscopic nodularity were both independent predictors of progression to EA. Not all HGD patients were treated with esophagectomy in this study and, therefore, the pos- sibility of undiagnosed EA could not be completely ruled out. ${ }^{61}$

In 2003, Dar et al. studied 42 patients treated with esophagectomy for a diagnosis of HGD. ${ }^{62}$ They defined focal HGD as dysplasia present at one level and diffuse HGD as dysplasia present at more than one level of the BE segment. ${ }^{62}$ The extent of HGD, as defined by their own criteria, and by those of Buttar et al., did not predict presence of EA in the esophagectomy specimen. ${ }^{61,62}$

Extent of both LGD and HGD as a predictor of progression to EA was analyzed in a more recent study by Srivastava et al. ${ }^{63}$ All biopsies were scored for the total number of crypts and the total number of crypts with LGD or HGD which provided a measure of percentage of dysplastic crypts with LGD or HGD in each biopsy. The primary endpoint of this retrospective longitudinal study was a histologic diagnosis of EA. The patients who progressed to cancer had a significantly higher mean percentage of total dysplastic crypts and crypts with LGD but not HGD. The authors also defined focal dysplasia as dysplasia present in one fragment, and diffuse dysplasia as dysplasia present in more than one fragment, regardless of the level of the $\mathrm{BE}$ segment. Using this definition, diffuse LGD or combined LGD and HGD was significantly associated with progression to EA. The focal and diffuse definitions of Buttar et al. and Dar et al., when applied to this data set, did not predict progression to EA.

In view of the conflicting data discussed earlier, an objective and standardized approach to evaluate the extent of dysplasia is needed. We favor abandoning the use of "focal" and "diffuse" dysplasia to assess extent of dysplasia because any proposed definition will be arbitrary. We propose that the extent of dysplasia be evaluated using a four part approach that will allow a more objective assessment of extent of dysplasia in patients with BE: (1) studies evaluating extent of dysplasia as a predictor of progression to EA should report the number of biopsies obtained from flat mucosa and those taken from areas of endoscopic abnormality to avoid a sampling bias. Estimation of dysplasia extent should be performed and reported separately for flat mucosa and endoscopic abnormality. (2) When only a single biopsy fragment is involved with dysplasia, the number and percentage of crypts with LGD, HGD, or combined LGD and HGD 
should be provided. (3) If more than one fragment from the same level is positive for dysplasia, then the total number of fragments, the number of fragments with dysplasia, and the grade(s) of dysplasia should be recorded. (4) Finally, the number of levels in a BE segment positive for dysplasia and the grade(s) of dysplasia should be recorded.

Using this approach, the data across relevant studies on this subject can be easily compared and allow correlation of accurately defined groups of extent of dysplasia with risk of progression to EA. This may eventually lead to detection of a threshold that enables meaningful stratification of BE patients with dysplasia into those at low and high risk of progression to EA.

\section{What are the differences between the main categorization systems used for grading dysplasia in BE?}

Robert H. Ridddell

rriddell@mtsinai.on.ca

It needs to be appreciated that these two systems were "invented" for completely different reasons. The IBD-C was created in the early 1980s to try to resolve numerous issues causing problems at that time. ${ }^{64}$ These included (1) no definition or criteria for dysplasia; (2) three grades of dysplasia in general use-mild, moderate, and severe, which may or may not include carcinoma in situ (CIS); (3) ambiguity of terminology in use because terms such as "dysplasia," "mild dysplasia," and "atypia" were all used for both reactive and neoplastic changes; (4) the clinical implications of these diagnoses were unclear; and (5) for all of these reasons there could be no attempt to determine reproducibility.

The resulting classification did the following: dysplasia was defined as an unequivocally neoplastic proliferation and any grade could give rise directly to an invasive carcinoma. The classification system included four categories, each of which also had clinical implications that, until recently, also applied to $\mathrm{BE}$, as follows:

- Negative: continue surveillance;

- Indefinite: early rebiopsy following $\mathrm{Rx}$ if possible;
- LGD: early rebiopsy or consider colectomy; and

- HGD: consider colectomy.

Note: There were only two grades of dysplasia, so that "mild," "moderate," and "severe" dysplasia ceased to exist, and were (and still are) now obsolete terms. It was also found to be fairly reproducible, especially when no or HGD were present.

The aim of the VCS was to develop a common worldwide terminology for gastrointestinal epithelial neoplasia. In practice, this expanded to become a classification and grading system for both dysplasia (noninvasive) and invasive neoplasia, whereas the concepts, principles, and definition of dysplasia system were accepted from the 1983 publication. Its objectives were to minimize the widely recognized discrepancies in interpretation of morphologic dysplasia between Western and Japanese pathologists; and to reach a uniform consensus on the nomenclature of neoplastic precursor lesions.

The resulting classification system was as follows ${ }^{65}$ with the IBD-C shown on the right.

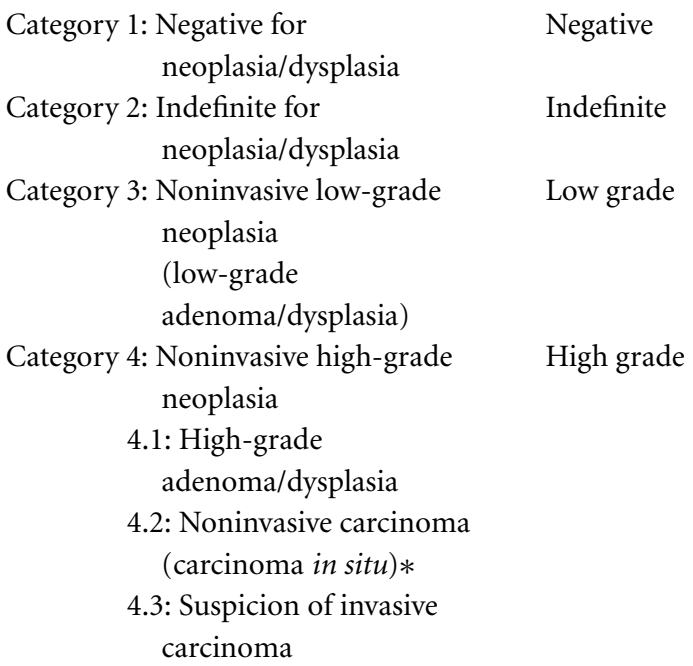

Category 5: Invasive neoplasia

5.1: Intramucosal carcinoma (lamina propria)

5.2: Submucosal carcinoma or beyond

In practice, there was a minor change in terminology, and the addition of several categories after high-grade dysplasia. 


\section{What can be expected from the endoscopic detection of dysplasia using 5-ALA sensitization and illumination with blue light?}

\section{Lawrence F. Johnson \\ lfjmd@bellsouth.net}

The compound 5-amino levulinic acid (5-ALA) is a prodrug that may be given orally or topically and is absorbed by the mucosa and metabolized to protoporphyrin IX (PpIX), a photo sensitizer. Because the degradation enzyme of PpIX, ferrochelatase, is deficient in dysplastic tissue, PpIX accumulates, and in blue light fluoresces. In turn, this fluorescence provides the endoscopist an opportunity to visually distinguish dysplastic tissue/adenocarcinoma with its intense fluorescence from that of normal tissue, and thereby, to perform target biopsies as opposed to random $4 \mathrm{QB}$ taken every one or two centimeters. ${ }^{66-68}$

This special fluorescent illumination was accomplished through a standard endoscope. To excite the PpIX, a filtered xenon lamp using blue light at a

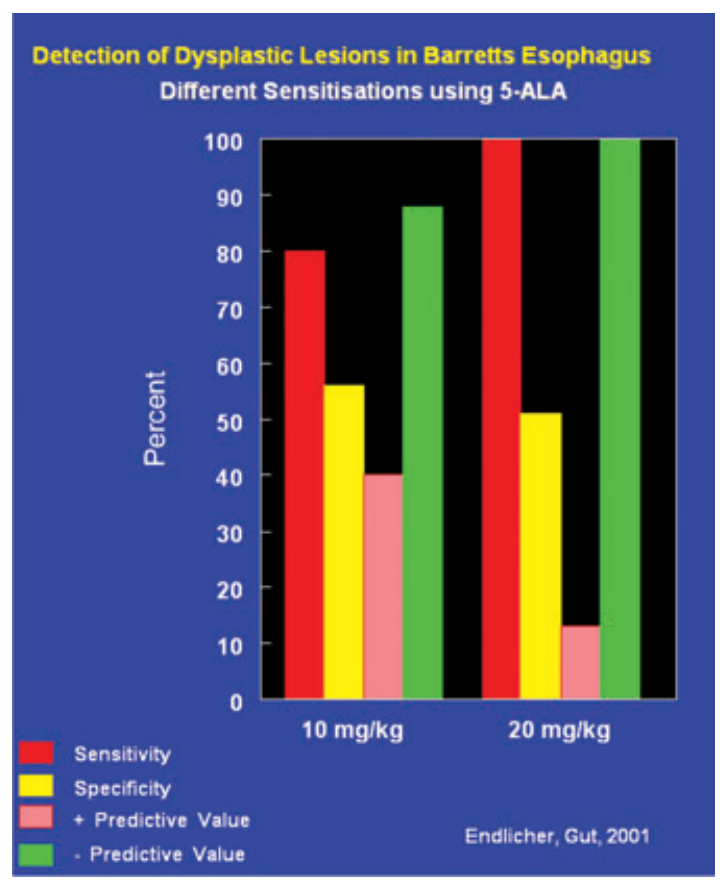

Figure 3. Sensitivity, specificity, and positive and negative predictive values determined from the presence or absence of dysplasia in fluorescent or nonfluorescent tissue using different doses of 5-ALA (from Endlicher et al. ${ }^{66}$ ). wave length of (380-460 nm) was connected to the scope, and in turn the ocular part of the scope was connected to a camera controller that sent either an image with white or the blue excitation light to the video monitor. ${ }^{68}$

The proof of the above concept was established by Endligher et al. ${ }^{66}$ who looked at sensitivity, specificity, and positive and negative predictive values determined from the presence or absence of dysplasia in fluorescent or nonfluorescent tissue using different doses of 5-ALA (Fig. 3). As shown, sensitivity occurred in the $80-100 \%$ range with the 10 and $20 \mathrm{mg} / \mathrm{kg}$, dose respectively, and specificity much less (56\% and 51\%, respectively). However, the negative predictive value was high in both groups (90-100\%, respectively). That is, if the mucosa was fluorescence-negative, no dysplasia was found-an endoscopically helpful and clinically relevant finding in the surveillance of patients with BE. Based on these results, the authors recommended a randomized prospective clinical trial to demonstrate the benefit of endoscopic FED compared to the standard technique of white light endoscopy with

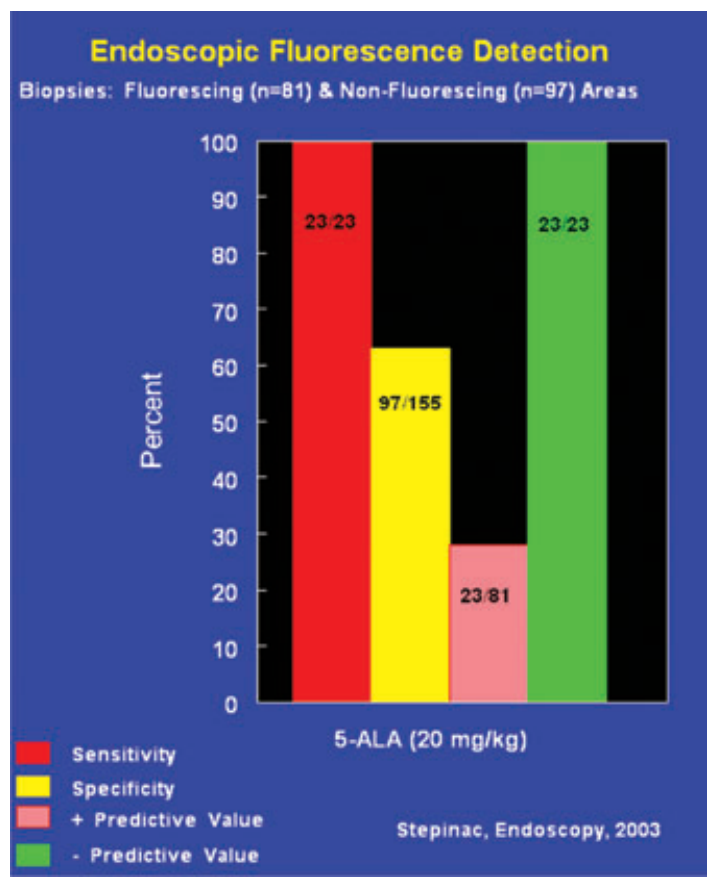

Figure 4. Comparable sensitivity, specificity, and positive and negative values were obtained for dysplasia found in fluorescing and nonfluorescing mucosal areas as reported by Endlicher et al. ${ }^{66}$ 


\section{Comparison (28 patients)}

\section{Four Quadrant Random Biopsies}

Endoscopic

Fluorescence Detection

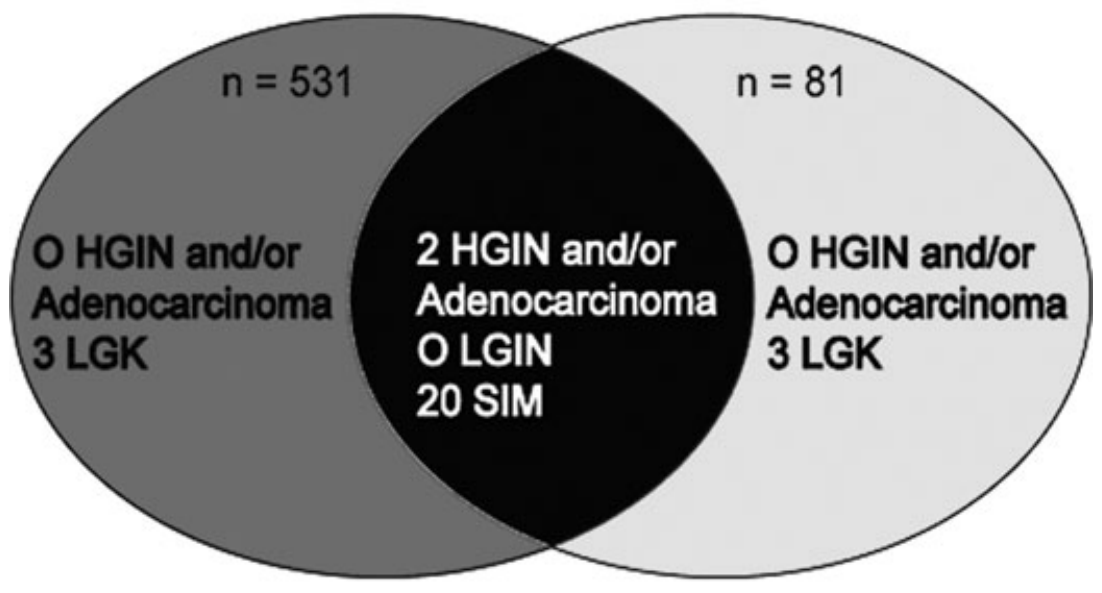

Stepinac, Endoscopy, 2003

\section{Figure 5.}

random biopsies taken every $1-2 \mathrm{~cm}$ in four quadrants in patients with $\mathrm{BE}$.

The above proposed randomized clinical trial was completed and published by Stepinac et al. ${ }^{68}$ In their study involving 28 patients, the authors used endoscopic fluorescence with 5-ALA to target biopsies for dysplasia and compare them to those obtained randomly using white light (four quadrants every $1 \mathrm{~cm}$ ). Again, comparable sensitivity, specificity, and positive and negative values were obtained for dysplasia found in fluorescing and nonfluorescing mucosal areas as reported by Endlicher et al. ${ }^{66}$ (Fig. 4). Most importantly, in the comparison between $4 \mathrm{QB}$ obtained every $1 \mathrm{~cm}$ and those that used endoscopic FED, both techniques gave comparable diagnostic accuracy (Fig. 5). However, endoscopic FED achieved that result with only 81 biopsies as opposed to 531 for the conventional white light technique that took random biopsies from fourquadrants every $1 \mathrm{~cm} .{ }^{68}$

Wanting to eliminate the human eye from qualitatively determining fluorescence versus nonfluo- rescence, Bland et al. ${ }^{2}$ used a laser spectrograph system that measured fluorescence intensity at $635 \mathrm{~nm}$ and compared both the presence or absence of dysplasia and fluorescence intensity from the mucosa immediately before the biopsies were obtained. As expected, high-grade dysplasia had protoporphyrin fluorescence intensity significantly greater than that found in non-dysplastic Barrett's epithelium. Interestingly, sometimes nodular dysplastic mucosa sometimes had less PpIX fluorescence intensity than the surrounding mucosa with high-grade dysplasia. This inconsistency in detection was overcome by establishing a fluorescence intensity ratio of 635 $\mathrm{nm} / 480 \mathrm{~nm}$, which permitted nodular high-grade dysplasia to be differentiated from non-dysplastic tissue with $100 \%$ sensitivity and specificity.

In summary, PpIX fluorescence appears useful for identifying areas of high-grade dysplasia so that target biopsies might be obtained in patients with BE. In support of this assertion, endoscopic FED gives a comparable result compared to that obtained with four-quadrants biopsies taken every $1 \mathrm{~cm}$; 
however, with fewer biopsies, and, by implication, less time.

\section{What is the technique that allows for best selection of biopsies and endoscopic diagnosis of dysplasia?}

\author{
Michael D. Saunders \\ mds@u.washington.edu
}

Current recommended endoscopic surveillance of BE using the Seattle protocol, 4QB taken every 1-2 cm of the Barrett's segment has limitations. These include difficulty in visualizing early neoplastic lesions with standard white light endoscopy, that random biopsies may sample on approximately $5 \%$ of the Barrett's mucosa, and that the dysplasia can have a patchy and focal distribution. ${ }^{69}$ These limitations have ignited a search for an adjunctive imaging tool to detect and target areas of dysplasia. Ideally, this tool or technique would reliably detect early neoplasia, decrease the already low pretest probability of dysplasia, and, in the absence of neoplasia, enable risk stratification comparable to current histology (i.e., no dysplasia versus low grade). ${ }^{69}$ To date, no technique has fulfilled these ideal criteria and, therefore, cannot yet replace the need for random 4QB. Advanced imaging techniques that have been evaluated for the detection of Barrett's dysplasia are summarized below with their respective advantages and current limitations (Table 5).

NBI and CLE have been shown to be more accurate than random biopsies, requiring fewer total biopsies, in detecting and targeting dysplasia in high-risk patients (those with known or suspected dysplasia). ${ }^{70,71}$ However, the overall yield of dysplasia per patient is not changed. Furthermore, the sensitivity of these techniques is decreased in patients at lower risk for dysplasia, who are the majority of Barrett's patients. ${ }^{70}$

Endoscopic trimodal imaging (ETMI), ${ }^{56,71,75}$ which includes HRE, autofluorescence (AF), and NBI has been recently compared to standard white light endoscopy with random 4QB in high-risk patients with suspected early Barrett's cancer and no endoscopically visible abnormality. ${ }^{72}$ No significant difference was observed in overall yield per patient of dysplasia. AF imaging improved targeted detection of early Barrett's cancer compared to standard endoscopy. NBI decreased false positives but was associated with a false negative rate of $17 \%$. Similar results were seen in a separate community-based study involving intermediate risk patients. ${ }^{73}$

\section{Summary}

HRE has likely become the default standard imaging technique for best selection of biopsies and endoscopic diagnosis of dysplasia. ETMI (AFI) and CLE improve the targeted detection of dysplasia in highrisk patients. However, no current technique can

Table 5. Advanced imaging techniques for detection of dysplasia in Barrett's esophagus

\begin{tabular}{ll}
\hline Imaging technique & Comment \\
\hline $\begin{array}{l}\text { High-resolution, white-light endoscopy (HRE) } \\
\text { Narrow band imaging (NBI) }\end{array}$ & $\bullet$ Becoming the standard \\
& $\bullet$ Yield similar to routine biopsies but with fewer biopsies \\
Autofluorescence imaging (AFI) & $\bullet$ Difficulty with pattern recognition \\
& $\bullet$ Improves detection of dysplasia \\
Endoscopic trimodal imaging (high resolution & $\bullet$ High false positive rates \\
endoscopy, autofluorescence, NBI) & $\bullet$ Improves targeted detection of dysplasia in high-risk patients \\
Confocal laser endomicroscopy (CLE) & $\bullet$ In vivo imaging with subcellular resolution of focal areas \\
Magnification endoscopy & $\bullet$ Image interpretation \\
Chomoendoscopy & $\bullet$ No comparisons with standard endoscopy \\
& $\bullet$ Tedious to use \\
& $\bullet$ Varying results in RCTs \\
& $\bullet$ Lack of standardization of technique
\end{tabular}

Adapted from Sharma et al. ${ }^{2}$ 
at this time replace random $4 \mathrm{QB}$, particularly in low-to-intermediate risk patients, for detection of dysplasia.

\section{What are the criteria to differentiate inflammatory or regenerative changes from dysplasia?}

\section{Ram Chuttani}

rchuttan@bidmc.harvard.edu

\section{Key issues}

- A wide spectrum of atypia exists in patients with Barrett's and persistent reflux.

- Regenerative changes may be extreme, especially in mucosa adjacent to the neo-SCJ or in areas of active inflammation and ulceration.

- These changes may lead to overdiagnosis of dysplasia. $^{74,75}$

\section{What is seen pathologically in inflammation?}

- Cytologic features of inflammation and preservation of crypt architecture. In severe inflammation-crypt budding, branching, atrophy, crowding, distortion, or even cystic changes may be seen.

- There may be nuclear stratification particularly at the bases of crypts, but occasionally also at the surface.

\section{Inflammation versus dysplasia}

- Regenerating cells contain nuclei with smooth membranes, normal nuclear/cytoplasmic ratio, and variable number of normal mitosis.

- Dysplastic cells show nuclear pleomorphism, loss of cell polarity, and markedly increased nuclear/cytoplasmic ratio.

- Regenerating cells contain nuclei with smooth membranes, normal nuclear/cytoplasmic ratio, and variable number of normal mitosis.

- Dysplastic cells show nuclear pleomorphism, loss of cell polarity, and markedly increased nuclear/cytoplasmic ratio.

\section{How to differentiate inflammation from dysplasia?}

- Although in inflammation, some changes of increased nuclear/cytoplasmic ratio, hyperchromacity, and slight loss of polarity and pleomorphism may be present, tufting of surface cells helps distinguish from dysplasia.
- In inflammation, goblet cells, both normal and dystrophic, are common.

- In inflammation, cytoplasmic mucin may be depleted but the crypt cells show a progressive increase in mucin close to or at the luminal surface.

- The surface maturation is exhibited by preservation of nuclear/cytoplasmic ratio and decrease in nuclear stratification in the upper level of the crypts.

- The degree of atypia related to regenerative changes decreases in mucosa more distant from the inflammation.

\section{Acknowledgments}

The authors thanks Professor Raj K. Goyal and Professor John Hayes for assistance of the manuscript preparation.

\section{Conflicts of interest}

The authors declare no conflicts of interest.

\section{References}

1. Armstrong, D. 2004. Review article: towards consistency in the endoscopic diagnosis of Barrett's oesophagus and columnar metaplasia. Aliment Pharmacol. Ther. 20(Suppl. 5): 4047.

2. Sharma, P., J. Dent, D. Armstrong, et al. 2006. The development and validation of an endoscopic grading system for Barrett's esophagus: the Prague C\&M criteria. Gastroenterology 131: 1392-1399.

3. Amano, Y., N. Ishimura, K. Furuta, et al. 2006. Which landmark results in a more consistent diagnosis of Barrett's esophagus, the gastric folds or the palisade vessels? Gastrointest. Endosc. 64: 206-211.

4. Ishimura, N., Y. Amano \& Y. Kinoshita. 2009. Endoscopic definition of esophagogastric junction for diagnosis of Barrett's esophagus: importance of systematic education and training. Digest Endsc. 21: 213-218.

5. Fléjou, J.F. \& M. Svrcek. 2007. Barrett's oesophagus-a pathologist's view. Histopathology. 50: 3-14.

6. Wang, K.K. \& R.E. Sampliner. 2008. Updated guidelines 2008 for the diagnosis, surveillance and therapy of Barrett's esophagus. Am. J. Gastroenterol. 103: 788-797.

7. Playford, R.J. 2006. New British Society of Gastroenterology (BSG) guidelines for the diagnosis and management of Barrett's oesophagus. Gut 55: 442-443.

8. Spechler, S.J. 1997. Short and ultrashort Barrett's esophagus-what does it mean? Semin. Gastrointest. Dis. 8: 59-67.

9. Rudoloph, R.E., T.L. Vaughan, M.E Storer, et al. 2000. Effect of segment length on risk for neoplastic progression in patients with Barrett esophagus. Ann. Intern. Med. 132: 612-620. 
10. Vahabzadeh, B., A.B. Seetharam, M.B. Cook, et al. 2010. Validation of the Prague C\&M criteria for the endoscopic grading of Barrett's esophagus among gastroenterology trainees: a multicenter study. Gastrointest. Endosc. 71: AB156.

11. Kinjo, T., C. Kusano, I. Oda, et al. 2010. Prague C\&M and Japanese criteria: shades of Barrett's esophagus endoscopic diagnosis. J. Gastroenterol. In press.

12. Ngamruengphong, S., V.K. Sharma \& A. Das. 2009. Diagnostic yield of methylene blue chromoendoscopy for detecting specialized intestinal metaplasia and dysplasia in Barrett's esophagus: a meta-analysis. Gastrointest. Endosc. 69: 10211028.

13. Guelrud, M. \& I. Herrera. 1998. Acetic acid improves identification of remnant islands of Barrett's epithelium after endoscopic therapy. Gastrointest. Endosc. 47: 512-515.

14. Guelrud, M., I. Herrera, H. Essenfeld \& J. Castro. 2001. Enhanced magnification endoscopy: a new technique to identify specialized intestinal metaplasia in Barrett's esophagus. Gastrointest. Endosc. 53: 559-565.

15. Curvers, W.L., F.J. Van Den Broek, J.B. Reitsma, et al. 2009. Systematic review of narrow-band imaging for the detection and differentiation of abnormalities in the esophagus and stomach. Gastrointest. Endosc. 69: 307-317.

16. Kiesslich, R., L. Gossner, M. Goetz, et al. 2006. In vivo histology of Barrett's esophagus and associated neoplasia by confocal laser endomicroscopy. Clin. Gastroenterol. Hepatol. 4: 979-987.

17. Harrison, R., I. Perry, W. Haddadin, et al. 2007. Detection of intestinal metaplasia in Barrett's esophagus: an observational comparator study suggests the need for a minimum of eight biopsies. Am. J. Gastroenterol. 102: 1154-1161.

18. Eloubeidi, M.A. \& D. Provencal. 1999. Does this patient have Barrett's esophagus? The utility of predicting Barrett's esophagus at the index endoscopy. Am. J. Gastro. 94: 937.

19. Hanna, S., A. Rastogi, A.P. Weston, et al. 2006. Detection of Barrett's esophagus after endoscopic healing of erosive esophagitis. Am. J. Gastro. 101: 1416-1420.

20. Koslowsky, B., H. Jacob, R. Eliakim, et al. 2006. PillCam ESO in esophageal studies: improved diagnostic yield of 14 frames per second (fps) compared with 4 fps. Endoscopy 38: $27-30$.

21. Eliakim, R., V.K. Sharma, K. Yassin, et al. 2005. A prospective study of the diagnostic accuracy of PillCam ESO esophageal capsule endoscopy versus conventional upper endoscopy in patients with chronic gastroesophageal reflux diseases. J. Clin. Gastroenterol. 39: 572-578.

22. Lin, O.S., D.B. Schembre, K. Mergener, et al. Blinded comparison of esophageal capsule endoscopy versus conventional endoscopy for diagnosis of Barrett's esophagus in patients with chronic gastroesophageal reflux. Gastrointest. Endosc. In press.

23. Gerson, L. \& O.S. Lin. 2007. Cost-benefit analysis of capsule endoscopy compared with standard upper endoscopy for the detection of Barrett's esophagus. Clin. Gastroenterol. Hepatol. 5: 319-325.

24. Rubenstein, J.H., J.M. Inadomi, J.V. Brill \& G.M. Eisen. 2007. Cost utility of screening for Barrett's esophagus with esophageal capsule endoscopy versus conventional upper endoscopy. Clin. Gastroenterol. Hepatol. 5: 312-318.
25. Gralnek, I.M., R. Rabinovitz, D. Afik \& R. Eliakim. 2006. A simplified ingestion procedure for esophageal capsule endoscopy: initial evaluation in healthy volunteers. Endoscopy 38: 913-918.

26. Gralnek, I.M., S.N. Adler, K. Yassin, et al. 2008. Detecting esophageal disease with second-generation capsule endoscopy: initial evaluation of the PillCam ESO 2. Endoscopy 40: 275-279.

27. Bhardwaj, A., C.S. Hollenbeak, N. Pooran \& A. Mathew. 2009. A meta-analysis of the diagnostic accuracy of esophageal capsule endoscopy for Barrett's esophagus in patients with gastroesophageal reflux disease. Am. J. Gastroenterol. 104: 1533-1539.

28. Ramirez, F.C., R. Akins \& M.R. Shaukat. 2008. Screening of Barrett's esophagus with string-capsule endoscopy: a prospective blinded study of 100 consecutive patients using histology as the criterion standard. Gastrointest. Endosc. 68: $25-30$.

29. Gerson, L.B. 2009. Screening for esophageal varices: is esophageal capsule endoscopy ready for prime time? J. Clin. Gastroenterol. 43: 899-901.

30. Ramirez, F.C., M.S. Shaukat, M.A. Young, et al. 2005. Feasibility of string wireless capsule endoscopy in diagnosisn Barrett's esophagus. Gastrointest. Endosc. 61: 641646.

31. Ramirez, F.C., Hakim S, E. Tharalson, et al. 2005. Feasibility and safety of string wireless capsule endoscopy in the diagnosis of esophageal varices. Am. J. Gastroenterol. 100: 1065-1071.

32. Sharma, P., N. Marcon, S. Wani, et al. 2006. Non-biopsy detection of intestinal metaplasia and dysplasia in Barrett's esophagus: a prospective multicenter study. Endoscopy 38: 120-1212.

33. Longcroft-Wheaton, G.A., M. Duku, R. Mead, et al. 2010. Acetic acid spray is an effective tool for the endoscopic detection of neoplasia in patients with Barrett's esophagus. Clin. Gastroenterol. Hepatol. 8: 843-847 [Epub 2010 Jun 30].

34. Curvers, W.L., R. Singh, M.B. Wallace, et al. 2009. Identification of predictive factors for early neoplasia in Barrett's esophagus after autofluorescence imaging: a stepwise multicenter structured assessment. Gastrointest. Endosc. 70: 9-17 [Epub 2009 Apr 25].

35. Curvers, W.L, L.A. Herrero, M.B. Wallace, et al. Endoscopic tri-modal imaging is more effective than standard endoscopy in identifying early-stage neoplasia in Barrett's esophagus. Gastroenterology 139: 1106-1114.

36. Pohl, J., O. Pech, A. May, et al. 2010. Incidence of macroscopically occult neoplasias in Barrett's esophagus: are random biopsies dispensable in the era of advanced endoscopic imaging? Am. J. Gastroenterol.

37. Kiesslich, R. \& H. Tajiri. 2010. Advanced Imaging in Endoscopy. In Gastroenterological Endoscopy. 2nd ed. M. Classen, G.N.J. Tytgat \& C.J. Lightdale, Eds.: 21-35. Thieme. Stuttgart/New York.

38. Elta, G. \& K.K, Wang, Eds. 2009. Enhanced endoscopic imaging. Gastrointest. Endosc. Clin. N. Am. 19: 193-314.

39. Norimura, D., H. Isomoto, T. Nakayama, et al. 2010. Magnifying endoscopic observation with narrow band imaging for specialized intestinal metaplasia in Barrett's esophagus 
with special reference to light blue crests. Dig. Endosc. 22: 101-106.

40. Mannath, J., V. Subramanian, C.J. Hawkey \& K. Ragunath. 2010. Narrow band imaging for characterization of high grade dysplasia and specialized intestinal metaplasia in Barrett's esophagus: a meta-analysis. Endoscopy 42: 351-359.

41. Kim, S.L., J.P. Waring, S.J. Spechler, et al. 1994. Diagnostic inconsistencies in Barrett's esophagus. Department of Veterans Affairs Gastroesophageal Reflux Study Group. Gastroenterology 107: 945-949.

42. Goda, K., H. Tajiri, M. Ikegami, et al. 2007. Usefulness of magnifying endoscopy with narrow band imaging for the detection of specialized intestinal metaplasia in columnarlined esophagus and Barrett's adenocarcinoma. Gastrointest. Endosc. 65: 36-46.

43. Wolfsen, H.C., J.E. Crook, M. Krishna, et al. 2008. Prospective, controlled tandem endoscopy study of narrow band imaging for dysplasia detection in Barrett's Esophagus. Gastroenterology 135: 24-31.

44. Sharma, P., A. Bansal, S. Mathur, et al. 2006. The utility of a novel narrow band imaging endoscopy system in patients with Barrett's esophagus. Gastrointest. Endosc. 64: 167-175.

45. Kara, M.A., M. Ennahachi, P. Fockens, et al. 2006. Detection and classification of the mucosal and vascular patterns (mucosal morphology) in Barrett's esophagus by using narrow band imaging. Gastrointest. Endosc. 64: 155-166.

46. Singh, R., G.K. Anagnostopoulos, K. Yao, et al. 2008. Narrow-band imaging with magnification in Barrett's esophagus: validation of a simplified grading system of mucosal morphology patterns against histology. Endoscopy 40: 457-463.

47. Curvers, W.L., F.J. ten Kate, K.K. Krishnadath, et al. 2010. Low-grade dysplasia in Barrett's esophagus: overdiagnosed and underestimated. Am. J. Gastroenterol. 105: 1523-1530.

48. Falk, G.W., R. Chittajallu, J.R. Goldblum, et al. 1997. Surveillance of patients with Barrett's esophagus for dysplasia and cancer with balloon cytology [see comments]. Gastroenterology 112: 1787-1797.

49. Pan, Q.J., M.J. Roth, H.Q. Guo, et al. 2008. Cytologic detection of esophageal squamous cell carcinoma and its precursor lesions using balloon samplers and liquid-based cytology in asymptomatic adults in Linxian, China. Acta. Cytol. 52: 14-23.

50. Saad, R.S., L.K. Mahood, K.M. Clary, et al. 2003. Role of cytology in the diagnosis of Barrett's esophagus and associated neoplasia. Diagn. Cytopathol. 29: 130-135.

51. Liu, S.F., Q. Shen, S.M. Dawsey, et al. 1994. Esophageal balloon cytology and subsequent risk of esophageal and gastriccardia cancer in a high-risk Chinese population. Int. JCancer 57: 775-780.

52. Fritcher, E.G., S.M. Brankley, B.R. Kipp, et al. 2008. A comparison of conventional cytology, DNA ploidy analysis, and fluorescence in situ hybridization for the detection of dysplasia and adenocarcinoma in patients with Barrett's esophagus. Hum. Pathol. 39: 1128-1135.

53. Reid, B.J., P.L. Blount, C.E. Rubin, et al. 1992. Flowcytometric and histological progression to malignancy in Barrett's esophagus: prospective endoscopic surveillance of a cohort. Gastroenterology 102(4 Pt 1): 1212-1219.
54. Sikkema, M., M. Kerkhof, E.W. Steyerberg, et al. 2009. Aneuploidy and overexpression of Ki67 and p53 as markers for neoplastic progression in Barrett's esophagus: a case-control study. Am. J. Gastroenterol. 104: 2673-2680.

55. Huang, Q., C. Yu, X. Zhang \& R.K. Goyal. 2008. Comparison of DNA histograms by standard flow cytometry and image cytometry on sections in Barrett's adenocarcinoma. BMC Clin. Pathol. 8: 5.

56. Shankey, TV., P.S. Rabinovitch, B. Bagwell, et al. 1993. Guidelines for implementation of clinical DNA cytometry. International Society for Analytical Cytology. Cytometry 14: 472-477.

57. Yu, C.G., Q. Huang, M. Klein \& R.K. Goyal. 2007. Quantitative targeted DNA index analysis in Barrett's mucosa, dysplasia and carcinoma. Lab. Invest. 87: 466-472.

58. Merlo, L.M., L.S. Wang, J.W. Pepper, et al. 2010. Polyploidy, aneuploidy and the evolution of cancer. Adv. Exp. Med. Biol. 676: $1-13$.

59. Rabinovitch, P.S., G. Longton, P.L. Blount, et al. 2001. Predictors of progression in Barrett's esophagus III: baseline flow cytometric variables. Am. J. Gastroenterol. 96: 3071-3083.

60. Yu, C., X. Zhang, Q. Huang, et al. 2007. High-fidelity DNA histograms in neoplastic progression in Barrett's esophagus. Lab. Invest. 87: 466-472.

61. Buttar, N.S., K.K. Wang, T.J. Sebo, et al. 2001. Extent of high-grade dysplasia in Barrett's esophagus correlates with risk of adenocarcinoma. Gastroenterology 120: 16301639.

62. Dar, M.S., J.R. Goldblum, T.W. Rice \& G.W. Falk. Can extent of high grade dysplasia in Barrett's oesophagus predict the presence of adenocarcinoma at oesophagectomy? Gut 52: 486-489.

63. Srivastava, A., J.L. Hornick, X. Li, et al. 2007. Extent of low-grade dysplasia is a risk factor for the development of esophageal adenocarcinoma in Barrett's esophagus. Am. J. Gastroenterol. 102: 483-493.

64. Riddell, R.H., H. Goldman, D.F. Ransohoff, et al. 1983. Dysplasia in inflammatory bowel disease: standardized classification with provisional clinical applications. Hum. Pathol. 14: 931-968.

65. Schlemper, R.J., R.H. Riddell, Y. Kato, et al. 2000. The Vienna classification of gastrointestinal epithelial neoplasia. Gut 47: 251-255.

66. Endlicher, E., R. Knuechel, T. Hauser, et al. 2001. Endoscopic fluorescence detection of low and high grade dysplasia in Barretts oesophagus using systemic or local 5aminolevulinic acid sensitization. Gut 48: 314-319.

67. Bland, S., T.D. Wang \& K.T. Schomacker. 2002. Detection of high-grade dysplasia in Barretts esophagus by spectroscopy measurement of 5-aminolevulinic acid-induced protoporphyrin IX fluorescence. Gastrointestinal. Endoscopy 56: 478487.

68. Stepinac, T., C. Felley \& P. Jornod. 2003. Endoscopic fluorescence detection of intraepithelial neoplasia in Barrett's esophagus after oral administration of aminolevulinic acid. Endoscopy 35: 663-668.

69. Bisschops, R. \& J. Bergman. 2010. Probe-based confocal laser endomicroscopy. Scientific toy or clinical tool? Endoscopy 42: 487-489. 
70. Sharma, P. 2009. Barrett's esophagus. N. Engl. J. Med. 361: 2548-2556.

71. Wallace, et al. 2010. Preliminary accuracy and interobserver agreement for the detection of intraepithelial neoplasia in Barrett's esophagus with probe-based confocal laser endomicroscopy. Gastrointest. Endosc. 72: 19-24.

72. Curvers, et al. 2010. Endoscopic tri-modal imaging is more effective than standard endoscopy in targeting earlystage neoplasia in Barrett's esophagus. Gastroenterology 139: 1106-1114.

73. Curvers, et al. 2011. Endoscopic trimodal imaging versus standard video endoscopy for detection of early Barrett's neoplasia: a multicenter, randomized, crossover study in general practice. Gastrointest. Endosc. 73: 195-203.

74. Odze, R.D. 2006. Diagnosis and grading of dysplasia in Barrett's esophagus. J. Clin. Pathol. 59: 1029-1038.

75. Goldblum, J.R. 2003.Barrett's esophagus and Barret's related dysplasia. Mod. Pathol. 16: 316-324.

76. Winters, C., T.C. Spurling, S.J. Chobanian, et al. 1987. Barrett's esophagus: A prevalent occult complication of gastroesophageal reflux disease. Gastroenterology 92: 118-124.

77. Cameron, A.J., C.T. Lomboy, M. Pera, et al. 1995. Adenocarcinoma of the esophagogastric junction and Barrett's esophagus. Gastroenterology 109: 1541-1546.

78. Johnston, M., A. Hammond, W. Laskin, et al. 1996. The prevalence and clinical characteristics of short segments of specialized intestinal metaplasia in the distal esophagus on routine endoscopy. Am. J. Gastroenterol. 1: 1507-1511.

79. Weston, A., P. Krmpotich, W. Makdisi, et al. 1996. Short segment Barrett's esophagus: Clinical and histological features, associated endoscopic findings, and association with gastric intestinal metaplasia. Am. J. Gastroenterol. 91: 981-986.

80. Trudgill, N.J., S.K. Suvarna, K.C. Kapur, et al. 1997. Intestinal metaplasia at the squamocolumnar junction in patients attending for diagnostic gastroscopy. Gut 41: 585-589.

81. Pereira, A.D., A. Suspiro, P. Chaves, A. Saraiva, L. Glória, J.C. de Almeida, C.N. Leitão, J. Soares, F.C. Mira. 1998. Short segments of Barrett's epithelium and intestinal metaplasia in normal appearing oesophagogastric junctions: the same or two different entities? Gut 42: 659-662.

82. Hackelsberger, T.G., G. Manes, J.E. Dominguez-Munoz, A. Roessner \& P. Malfertheiner. 1998. Intestinal metaplasia at the gastroesophageal junction: Helicobacter pylori gastritis or gastrooesophageal reflux disease? Gut 43: $17-21$.

83. Hirota, W.K., T.M. Loughney, D.J. Lazas, C.L. Maydonovitch, V. Rholl \& R.K.H. Wong. 1999. Specialized intestinal metaplasia, dysplasia and cancer of the esophagus and esophagogastric junction: prevalence and clinical data. Gastroenterology 116: 277-285. 